\title{
A review of surface effects in Kapitza's experiments on heat transfer between solids and helium II
}

\author{
(Review Article) \\ Jay Amrit \\ Laboratoire d'Informatique pour la Mécanique et les Sciences de l'Ingénieur, LIMSI-CNRS UPR 3251 \\ Université Paris Sud, Rue John von Neumann, 91405 Orsay, France \\ E-mail: jay.amrit@limsi.fr
}

Received April 1, 2016, published online June 25, 2016

\begin{abstract}
In a recent paper, it is shown that the thermal boundary Kapitza resistance between a solid and superfluid helium is explained by resonant scattering of phonons from surface roughness heights, as described in the Adamenko and Fuks (AF) model. We reexamine the original experiments of thermal transfer between a solid (platinum and copper) and superfluid helium conducted by P.L. Kapitza in 1940. In particular, we analyze his experimental results for the different surface treatments of the solid in light of the AF model. Time scales for diffuse scattering of phonons at the interface are estimated. Also the role of a layer of varnish on a copper surface is reinterpreted.
\end{abstract}

PACS: 62.60.+v Acoustical properties of liquids;

63.20.kp Phonon-defect interactions.

Keywords: Kapitza thermal resistance, solid-helium interfaces.

\section{Contents}

1. Introduction: “Anomalous” thermal boundary Kapitza resistance ...................................................787

2. A brief review Kapitza’s investigations of heat transfer between solids and helium II ..........................788

3. From acoustic mismatch to resonant phonon scattering ................................................................. 788

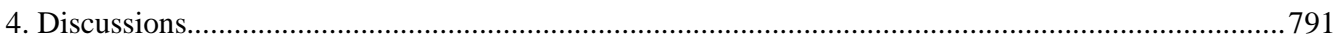

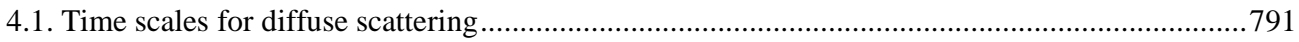

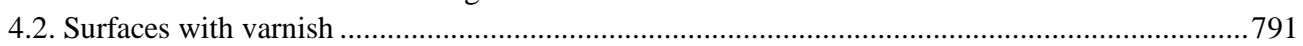

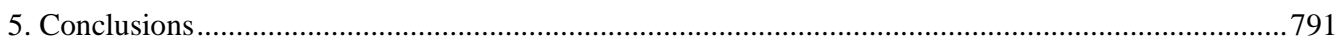

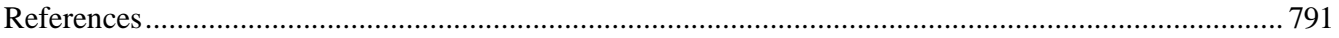

\section{Introduction: “Anomalous” thermal boundary Kapitza resistance}

In a paper published in the Journal of Physics in 1941 [1], P.L. Kapitza presented a series of experiments that he had conducted to study heat transfer in helium II. All these experiments were conducted within the two years of the discovery of superfluid helium (helium II) in 1938. Kapitza defined the heat conductivity across a solid into helium II as $m=k / h=(\dot{q} / \Delta T)$, where $k$ is the hypothetical "thermal conductivity" of some layer of thickness $h$ over which the temperature jump $\Delta T$ is measured when a well-known heat flux $\dot{q}$ is applied. The inverse of $m$ is now known as the Kapitza thermal boundary resistance $R_{K}=\Delta T / \dot{q}$.
It is interesting to note that Kapitza had immediately suspected that the surface state of the solid could play a decisive role in creating such a temperature jump. And, in order "to ascertain more completely the influence of the state of the surface a series of experiments were made in which the condition of the surface was treated in different ways" [1].

Almost ten years after Kapitza's findings, Khalatnikov formulated the acoustic mismatch (AM) model [2] and showed that the thermal boundary resistance is a general phenomenon occurring at all interfaces and at all temperatures due to a difference between the bulk acoustic impedance (product of the sound velocity and density) of each medium. However, the AM theory falls short in explaining 
Kapitza's thermal resistance measurements. Rather strangely, this led to Kapitza's experimental observations of the thermal boundary resistance between solids and helium II at $T>1 \mathrm{~K}$ to be classified as the "anomalous Kapitza resistance".

In 1971 Adamenko and Fuks formulated a theory in which phonons incident from liquid helium II onto a solid surface undergo resonant scattering due to surface roughness [3]. This theory is recently demonstrated in Ref. 4.

In this paper we give a brief review of Kapitza's original experiments. Then we present an analysis of his experimental data in light of the AF model to characterize the role of the surface roughness. The results of varnish on copper surfaces are examined and alternative explanations are suggested. Time scales for phonon transmission at the interface are estimated.

\section{A brief review Kapitza's investigations of heat transfer between solids and helium II}

Kapitza's first investigation of heat transfer between a solid and helium II was conducted using a specially designed apparatus which was hung freely in helium II. The apparatus consisted of a narrow glass tube, of length $6.5 \mathrm{~cm}$ and of diameter $1.85 \mathrm{~mm}$. A bronze wire, which served as a thermometer, was wound into a long spiral and placed inside the glass tube. A lead tube was placed around the thermometer inside the glass tube. The glass tube was filled with helium gas and hermetically sealed. The walls of the glass tube were covered by a thin layer of platinum which served as a heater. The use of superconducting lead tube to shield the thermometer from the magnetic field created by the current in the heater, suppressed perturbations in the thermometer readings. These precautions lead the bronze thermometer to attain a remarkable precision of $3.4 \mu \mathrm{K}$. The Pt heater was subjected to various heat loads and the resulting temperature differences (of the order of 1-7 $\mathrm{mK}$ ) were measured at a constant temperature of the helium bath. The experiments were performed between 1.6 and $2.1 \mathrm{~K}$ and at five different bath temperatures. The experimental results (diamonds) for platinum are shown in Fig. 1 and the fit through the data is given by $R_{K}=17.87 T^{-3.206} \mathrm{~cm}^{2} \cdot \mathrm{K} / \mathrm{W}$.

Kapitza designed a second series of experiments to investigate the influence of solid surface non-ideality on the temperature jump. They were conducted with well annealed pure copper. The copper sample was a parallelepiped with a bronze thermometer and a bifilar wound heater placed in situ. Kapitza found that the thermal boundary resistance remained unaltered when a copper surface was gold plated. This led him to conclude that the presence of oxides did not have a substantial effect.

In Fig. 1 we show the thermal boundary resistance for the polished copper surface (squares) which behaves as $R_{K}=6.24 T^{-2.105} \mathrm{~cm}^{2} \cdot \mathrm{K} / \mathrm{W}$. When the same polished cop-

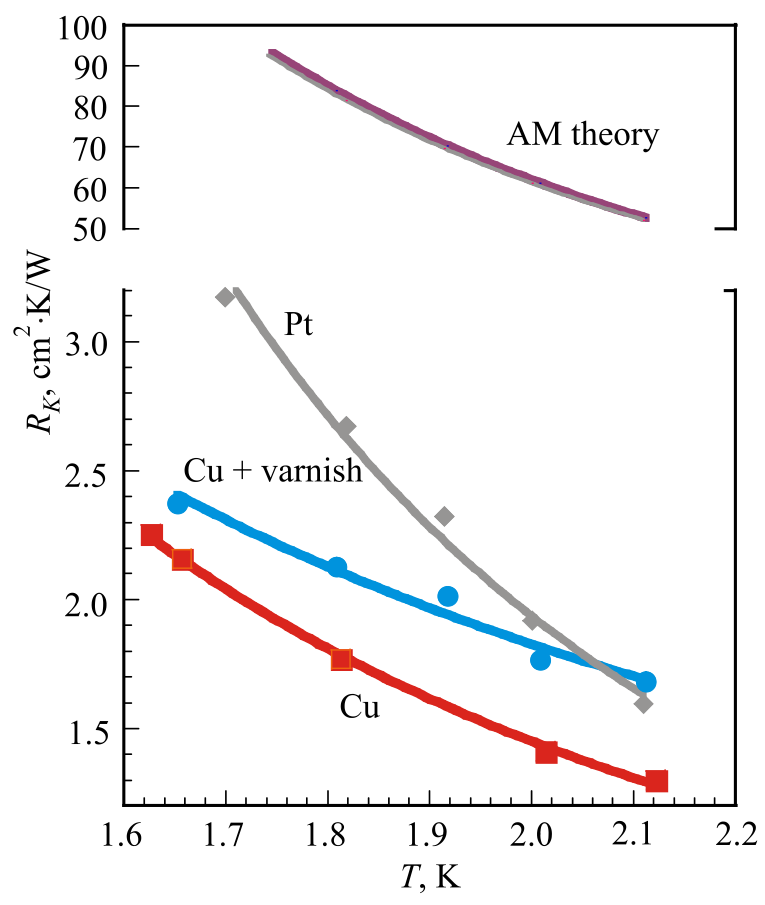

Fig. 1. Kapitza's measurements of heat transfer expressed as thermal boundary resistance for three different samples, namely polished $\mathrm{Cu}$, polished Cu sample covered with a layer of varnish, and a Pt sample. The AM theory predictions for $\mathrm{Cu}$ and $\mathrm{Pt}$ are almost identical and thereby support the hypothesis of bulk properties playing a secondary role in heat transfer between solids and helium II.

per surface was covered with a layer of varnish (matolin ${ }^{\circledR}$ ) (dots), the thermal boundary resistance increased and is given by $R_{K}=4.97 T^{-1.442} \mathrm{~cm}^{2} \cdot \mathrm{K} / \mathrm{W}$. Kapitza surmised that the increase in the thermal boundary resistance is due to the increase in the smoothness of the copper surface with a coat of varnish.

Finally, we remark that Kapitza concluded that the thermal boundary resistance follows a $T^{-\approx 3}$ behavior. From the fits in Fig. 1 we see that his results do not fully support this conjecture. We recall that these power law dependencies are not given in the Ref. 3.

\section{From acoustic mismatch to resonant phonon scattering}

We recall that in the Khalatnikov acoustic mismatch theory, the temperature jump at the interface is due to stringent conditions imposed by the conservation of frequency and the conservation of the parallel components of the momentum of phonons of each medium at the interface. These conditions define a cone of critical angle $\theta_{c}=\arcsin \left(c_{l} / c_{s}\right)$ in the liquid helium, where $c_{l}, c_{s}$ are, respectively, the sound velocities in helium II and in the solid, and generally $\theta_{c} \approx 3-4^{\circ}$. Only phonons within this cone have a non-zero probability of being transmitted into the solid. 
In Fig. 1 the AM theory prediction for copper and platinum is plotted using the expression

$$
R_{K 0}=\frac{30 \hbar^{3} \rho_{s} c_{t, s}^{3}}{\pi^{2} k_{B}^{4} \rho_{l} c_{l} F T^{3}},
$$

where $\hbar$ is the Planck's constant, $k_{B}$ is the Boltzmann constant, $\rho_{s}$ and $\rho_{l}$ are, respectively, the solid and liquid helium densities and $c_{t, s}$ is the transverse velocity in the solid. A particularly interesting point is that $R_{K 0} \cong 490 / T^{3} \mathrm{~cm}^{2} \cdot \mathrm{K} / \mathrm{W}$ is the same for both the copper and platinum immersed in helium II since the ratio $\left(\rho_{\mathrm{Cu}} c_{t, \mathrm{Cu}}^{3}\right) /\left(\rho_{\mathrm{Pt}} c_{t, \mathrm{Pt}}^{3}\right)=1.01$, with $\rho_{\mathrm{Cu}}=8.954 \mathrm{~g} / \mathrm{cm}^{3}, c_{t, \mathrm{Cu}}=2325 \mathrm{~m} / \mathrm{s}, \rho_{\mathrm{Pt}}=21.45 \mathrm{~g} / \mathrm{cm}^{3}$ and $c_{t, \mathrm{Pt}}=1730 \mathrm{~m} / \mathrm{s}$. Further, the factor $F$, which depends on the ratio of longitudinal to transverse velocities in each material, is almost identical for both the $\mathrm{Cu}$ and Pt samples and $F \cong 1.7$ according to Challis et al. [5]. Thanks to these coincidences (as there is no evidence to believe that Kapitza chose these elements on purpose), a comparison of all curves in Fig. 1 clearly suggests that some other mechanism related to the structure of solid surface plays a dominant role, independent of the bulk acoustic impedances.

The Adamenko and Fuks (AF) model examines the effect of small scale surface roughness on phonon transmission from helium II into the solid. More complex boundary conditions which take into account surface roughness are impose at the interface, and this, in some ways, relaxes the constraints imposed by the AM model at the interface. The transmission of phonons incident from helium II now depends on the surface roughness characteristics, namely the roughness height $\sigma$ and the roughness correlation length $\ell$. AF show that when phonon wavelengths are comparable to the correlation lengths of the roughnesses, phonons undergo resonant scattering and eventually diffract into the solid, carrying energy into it. This creates an "amplification" of the heat across the interface. Since the dominant phonon wavelength in helium II is $\lambda=h c_{L} /\left(3.83 k_{B} T\right) \approx(3 / T) \mathrm{nm}$, the roughnesses which play a predominant role are therefore nanometric in size. The thermal boundary resistance predicted by the AF model takes the form below when normalized with respect to the AM model:

$$
\frac{R_{K 0}}{R_{\zeta}}=1+57.75 \gamma^{2}\left(\frac{\ell}{\lambda}\right)^{2}
$$

where $\gamma=2 \sigma / \ell$ represents the inclination of a roughness. The numerical coefficient is due to constants. The amplification of the heat flux is determined by $\gamma$ and the ratio $\ell / \lambda$ independently.

Figure 2 represents the $\gamma$ values as a function of temperature for Kapitza's data given in Fig. 1. In calculating these $\gamma$ values we considered the case when the resonant scattering is a maximum, that is, when $\ell / \lambda \approx 1 / 3$ as defined

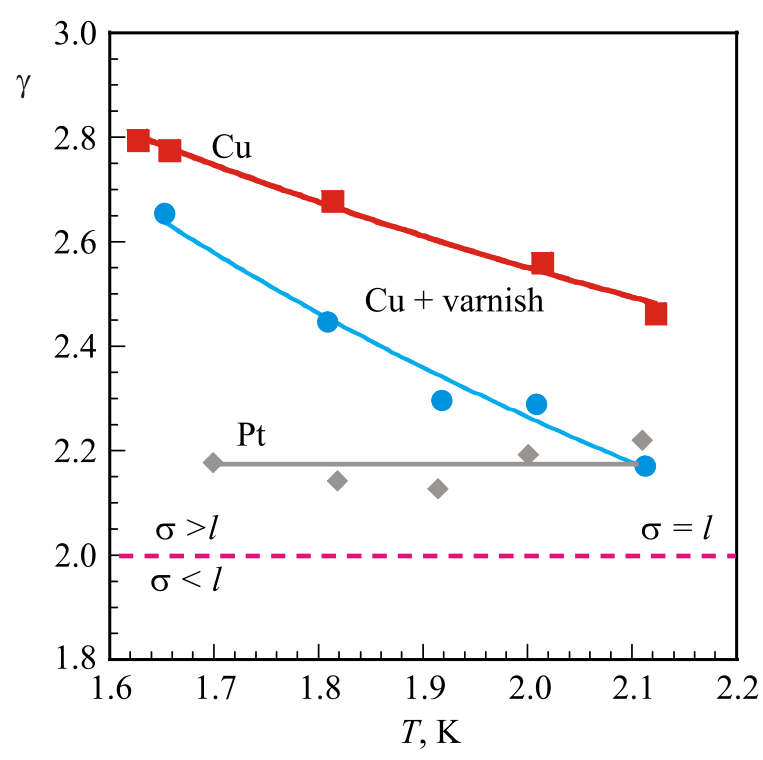

Fig. 2. Surface inclination $\gamma=2 \sigma / \ell$ determined as a function of temperature using Eq. (1), for the three different samples. For all samples the roughness height $\sigma$ is greater than the height-height correlation length $\ell$. The dashed line corresponds to $\sigma=\ell$. The fit to the Pt sample shows that $\gamma$ is independent of the temperature, whereas $\gamma$ decreases with temperature for the other $\mathrm{Cu}$ samples.

in the AF model. In Fig. 2, $\gamma>2$ indicates that the roughness height is slightly greater than their correlation length for all samples. For both the Cu samples, we see that as the temperature increases, $\lambda$ decreases and the roughness correlations lengths $\ell$ which come into play decrease. Consequently, $\sigma$ and $\gamma$ decrease with temperature. For the Pt sample, the $\gamma$ values remain constant with temperature. The fit to the Pt data in Fig. 3 gives $\sigma \approx 0.36 \lambda$ at all temperatures, suggesting the presence of a Gaussian distribution of roughnesses, according to the AF theory. This means that for the Pt sample the roughnesses are uniform with $\sigma \approx \ell$, whereas for the $\mathrm{Cu}$ samples, without and with varnish we have, respectively, $\sigma \approx 1.75 \ell^{1.46}$ and $\sigma \approx 1.97 \ell^{1.80}$. The power law dependencies indicate heterogeneity in the surface roughness of the $\mathrm{Cu}$ sample. In all the samples the different $\sigma$ values which participate and render the scattering resonant, depend on the temperature since $\lambda \propto T^{-1}$.

In Fig. 3 we show the roughness heights $\sigma$ as function of the dominant thermal wavelength at different temperatures. For the $\mathrm{Cu}$ sample $0.58 \mathrm{~nm} \leq \sigma \leq 0.86 \mathrm{~nm}$ whereas for the platinum sample $0.51 \mathrm{~nm} \leq \sigma \leq 0.64 \mathrm{~nm}$. The discrepancies among these values are small, but their impact on phonon transmission and on the temperature dependency is decisive. We note firstly that no other theory up to now, besides the AF theory, is capable of describing the various experimentally observed temperature dependencies of $R_{K}$. Secondly, Kapitza had suspected a strong influence of the solid surface state and had designed his experiments to investigate this aspect, as mentioned before. However it 


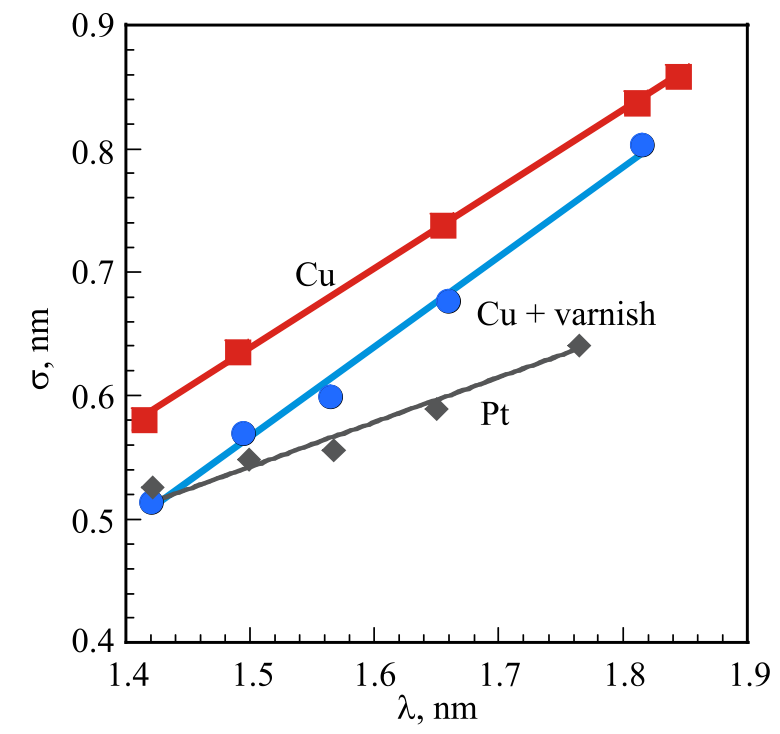

Fig. 3. Surface roughness height $\sigma$ as a function of dominant phonon wavelength $\lambda$ of He II. For the Pt sample, $\sigma \approx 0.36 \lambda$. Power law dependencies are found between $\sigma$ and $\lambda$ for the $\mathrm{Cu}$ and the varnished $\mathrm{Cu}$ samples, indicating these samples to have steep roughness heights.

is highly unlikely that he suspected roughnesses at a nanometric scale to play a key role in the origin of the thermal jump at the interface.

We shall now consider the influence of varnish on the $\mathrm{Cu}$ surface. The Kapitza resistance of the polished $\mathrm{Cu}$ surface with the varnish was measured to be higher than that of the polished $\mathrm{Cu}$ surface, as shown in Fig. 1. Kapitza suggested that the small difference between the two samples "can be accounted for by the increase in the smoothness by varnishing the surface and is not due to the thermal insulation properties of the film of varnish itself' [1]. It is well-known that varnish on a surface improves smoothness and therefore increases its gloss. The prospect of modifying the surface roughness height distribution with varnish is also interesting for technological reasons. In light of the AF model and as shown in Fig. 3, the roughness heights for the Cu surface with varnish vary as $0.51 \mathrm{~nm} \leq \sigma \leq 0.81 \mathrm{~nm}$. The difference in the roughness heights which participate in resonant scattering, between the $\mathrm{Cu}$ and the varnished $\mathrm{Cu}$ samples is less than $0.1 \mathrm{~nm}$. It is plausible for varnish of a few $\mu \mathrm{m}$ thick to have such an effect, especially if the surface roughness heights are greater than the correlation length of roughnesses, as is the case here.

We give here an alternate explanation and show that the discrepancy between $R_{K}$ values obtained for the bare $\mathrm{Cu}$ sample and the $\mathrm{Cu}$ with varnish sample (see Fig. 1) is primarily due to the thermal impedance of the varnish layer. We recall that the thermometer is placed inside the copper sample. The temperature jump measured by this thermometer includes effects due to the thermal impedance of the copper wall thickness and that of the varnish layer. Since the thermal conductivity of copper is five orders of magnitude greater than the thermal conductivity of varnish, the effect of the thermal impedance of the copper wall is completely negligible. The thickness of the varnish was estimated by Kapitza to be $e=3.4 \cdot 10^{-3} \mathrm{~mm}$. This thickness is equivalent to approximately 2000 times the thermal wavelength of a phonon in helium II in the temperature range of the experiment. Using a thermal conductivity for the varnish to be $K_{V}=7.6 \cdot 10^{-4} T^{0.8} \mathrm{~W} /(\mathrm{cm} \cdot \mathrm{K})$, the thermal impedance of the varnish layer given by $R_{V}=e / K_{V}$ turns out to make a significant contribution as illustrated in Fig. 4. The magnitude and the temperature dependency of our $K_{V}$ is in very good agreement with that measured in Ref. 6 for GE varnish No. 7031. The dashed line in the Fig. 4 corresponds to the $R_{K}$ of the $\mathrm{Cu}$ sample with a varnish layer after correcting for the thermal impedance of the varnish layer. This dashed line is in good agreement with the measured curve of $R_{K}$ for the $\mathrm{Cu}$ sample. The small discrepancy between these curves can be due to slight modifications in the surface roughness at nanoscales due to the varnish. The change in the surface roughness can also very well account for the difference in the temperature dependencies of $R_{K}$ for these samples according to the AF model. We also note that this simple analysis supposes a very strong bonding between the varnish and the copper solid surface. Above all this analysis supports the idea that resonant scattering as described by the AF model (second term in Eq. (1)) is independent of bulk properties of the solid in contact with helium II.

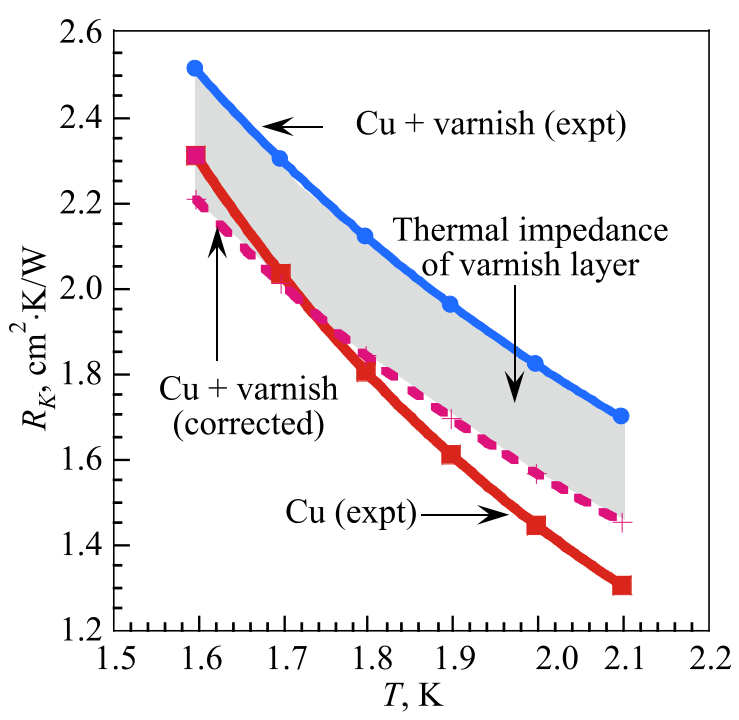

Fig. 4. The experimental $R_{K}$ of the $\mathrm{Cu}$ with varnish sample is "corrected" after removing the contribution of thermal impedance of varnish (shaded region) present in the measurement. The thermal conductivity of varnish $K_{V}=7.6 \cdot 10^{-4} T^{0.8} \mathrm{~W} /(\mathrm{cm} \cdot \mathrm{K})$. The $R_{K}$ values of the "corrected" $\mathrm{Cu}$ sample with varnish (dashed line) and that of the $\mathrm{Cu}$ sample are now very close, showing no significant change due to the presence of the varnish. 


\section{Discussions}

\subsection{Time scales for diffuse scattering}

Within a short space of time upon discovering the temperature jump, P.L. Kapitza conducted a series of investigations with $\mathrm{Cu}$, having polished surfaces, mat surfaces, dirty surfaces, surfaces with oxides, gold plated surfaces, surfaces with a layer of varnish to reduce roughness heights and surfaces covered with oil or emery powder. All these studies led to the conclusion that the surface state has an influence on the thermal boundary resistance and yield different temperature dependencies of $R_{K}$, depending on the surface treatment. Also, the $R_{K}$ values for all $\mathrm{Cu}$ samples with the different surface conditions differed by a factor of 5 at most, in the temperature range of 1.5 to $2.1 \mathrm{~K}$.

The Khalatnikov (AM) model cannot explain these discrepancies since the interface structure is absent in the model. In other words, the solid surface is ideally flat. The consequences of this aspect of the AM model can also be viewed as follows. In the AM model, if the time scale in which energy is removed at the interface is infinitely small at all temperatures, then the interface morphology shall not influence phonon transmission. To get some idea of the time scale of microscopic processes at the interface, we define the transmission of a phonon from liquid helium to occur over a duration $t \approx \sigma / c_{l}$, where $\sigma$ is the roughness height which comes into play in the scattering mechanism as defined in the AF model, and $c_{l}$ is the phonon speed in liquid helium. Diffuse resonant scattering occurs for a sample with an isotropic distribution of small scale roughnesses when we have $\sigma \approx \lambda / 3$, as in the case of $\mathrm{Pt}$ sample. Now, the time scale of phonon transmission becomes temperature dependent and is given by $t(T) \approx 4.2 / T$ ps, where the phonon wavelength in helium is $\lambda=(3 / T) \mathrm{nm}$ and $c_{l}=239 \mathrm{~m} / \mathrm{s}$. So in the temperature range of Kapitza's experiments discussed above $(1.5-2.1 \mathrm{~K})$, the typical time scale of microscopic transmission at the interface lies between 2-3 ps. During the transition from diffuse to specular scattering at the temperature decreases, the time scales must gradually decrease. This can be seen by rewriting $t \approx \frac{\sigma}{c_{l}}=\left(\frac{\sigma}{\lambda}\right) \frac{h}{3.83 k_{B} T}=\frac{12.54}{T}\left(\frac{\sigma}{\lambda}\right)$ ps. As specular scattering becomes predominant at low temperatures, we have from AF theory that $\sigma / \lambda \rightarrow 0$ and consequently $t \rightarrow 0$. Thus specular scattering occurs at time scales much smaller than a picosecond.

\subsection{Surfaces with varnish}

Although there is a substantial amount of experimental data [7] on $R_{K}$ accumulated over the years, investigations showing the influence of an ultra-thin layer of varnish on a solid surface in contact with liquid helium II have not been reiterated, to our recent knowledge, since Kapitza's first experiments.

It would be interesting to conduct these experiments with well qualified layers of varnish on a dielectric materi- al. The presence of varnish does indeed make a surface smoother and glossy in appearance. But this is due to surface roughness modifications at a scale of wavelengths of visible light which is of the order of $0.1 \mu \mathrm{m}$. Our analysis using the AF model above shows that at nanoscale levels, the surface roughness heights between the polished $\mathrm{Cu}$ and varnished $\mathrm{Cu}$ surface differ by $0.1 \mathrm{~nm}$. The effect of varnish in modifying the $R_{K}$, apart from the impedance effect due its thickness, needs to be confirmed. Secondly, varnish is a mixture of oil, resin and a solvent. Depending on its thickness and uniformity, it can very well be assimilated with "dirt” or non-metallic impurities [8]. Examining varnish in this light is also useful for technology applications. The general consensus from experiments is that the presence of impurities, even due to exposure to air (oxides) [9], dislocations [10] or "dirt" at the solid surface can no longer be considered to play predominant roles in explaining the anomalous Kapitza resistance. On the contrary, experiments, including those of Kapitza, show that these factors tend to increase the thermal boundary resistance.

\section{Conclusions}

P.L. Kapitza had designed his experiments to investigate the influence of surface conditions on heat transfer. In particular, his aim appears to be a search for a relationship between convection in helium II and the temperature jump close to solid wall. Ten years after his experiments on platinum and copper, the Khalatnikov (AM) model predicted for these samples to have very similar thermal resistances with helium II due to their almost identical bulk thermal impedances. This coincidence posteriorly supports the idea that the surface condition does indeed play a predominant role. Also, analyzing Kapitza results with the Adamenko and Fuks model does indeed corroborate the importance of surface roughness effects, but at scale lengths of a nanometer. The impact of varnish surface finish still needs to be fully studied in a larger temperature range. Finally we remark that initiating the investigations on the role of varnish surface finish and of the surface roughness in general, was particularly visionary of P.L. Kapitza.

1. P.L. Kapitza, J. Phys. 4, 181 (1941).

2. I.M. Khalatnikov, An Introduction to the Theory of Superfluidity, Addisson-Wesley, New York (1988).

3. I.N. Adamenko and I.M. Fuks, Sov. Phys. JETP 32, 1123 (1971).

4. A. Ramiere, S. Volz, and J. Amrit, Nat. Mater. 15, 512 (2016).

5. L.J. Challis and J.D.N. Cheeke, Proc. R. Soc. London 304, 479 (2012).

6. J.H. McTaggart and G.A. Slack, Cryogenics 9, 384 (1969).

7. E.T. Swartz and R.O. Pohl, Rev. Mod. Physics 61, 605 (1989).

8. H.J. Maris, Phys. Rev. B 19, 1443 (1979).

9. J. Weber, W. Sandmann, W. Dietsche, and H. Kinder, Phys. Rev. Lett. 40, 1469 (1978).

10. J. Amrit and A. Ramiere, Fiz. Nizk. Temp. 39, 970 (2013) [Low Temp. Phys. 39, 752 (2013)]. 